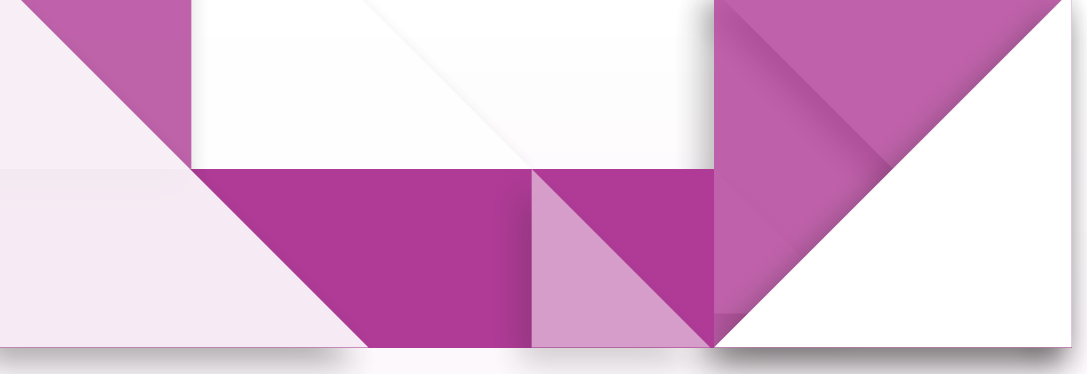

\title{
Diseño de una progresión de aprendizaje hipotética para la enseñanza de la estequiometría por comprensión conceptual e integrada
}

\section{- Design of a Hypothetical Learning Progression for Teaching Stoichiometry through Conceptual and Integrated Understanding}

- Elaboração de uma Trajetória Hipotética de Aprendizagem para o ensino da estequiometria por compreensão conceitual e integrada

\section{Resumen}

La estequiometría ha sido reconocida como uno de los temas de la química que implica mayor dificultad para su aprendizaje en los estudiantes de educación media e incluso en la educación universitaria. Una de las principales causas está en la falta de coherencia de los materiales instruccionales, los cuales en muchas ocasiones no consideran aspectos fundamentales como los conocimientos que son requisito, el lenguaje multinivel y las concepciones alternativas. Como propuesta para comenzar a superar la falta de coherencia intraunidad en los materiales instruccionales, se plantea el diseño de una progresión de aprendizaje hipotética (HLP) para la enseñanza de este tema. Se utiliza una metodología de perspectiva cualitativa, con la que se desarrolla un proceso sistemático de análisis documental a un conjunto de artículos, capítulos de libros, lineamientos curriculares y estándares básicos de competencias nacionales e internacionales relacionados con las progresiones de aprendizaje y la estequiometría, el cual tuvo lugar en dos fases. La primera, enfocada en elucidar los principales elementos teóricos y metodológicos que subyacen a este enfoque de diseño, construyendo además el marco conceptual de esta investigación. La segunda, centrada en la descripción del proceso de diseño de la HLP de la estequiometría tomando como referencia los principios de diseño y las secciones que configuran la estructura de la HLP. Como resultado del proceso de investigación se lograron tres aportes principales: marco teórico para la HLP desde la perspectiva de los estudios de diseño, desarrollo de los ítems de la HLP que generó las teorías de dominio específico para la enseñanza-aprendizaje de la estequiometría, y el material instruccional en formato digital. Se concluye que hoy las HLP son consideradas un paradigma emergente dentro del campo de la educación en ciencias, resultando ser una heurística exitosa con miras a producir teorías de dominio específico y materiales instruccionales con coherencia curricular.

Palabras clave

progresión de aprendizaje hipotética (HLP), estequiometría, coherencia, grandes ideas de la ciencia.
Docente del Instituto de Educación y Pedagogía, Universidad del Valle.

bofeca@yahoo.com

Orcid 0000-0002-5833-1975<? >

** Estudiante de Maestría en Educación, con énfasis en Enseñanza de las Ciencias Naturales de la Universidad del Valle.

roosvelt7@gmail.com

Orcid: 0000-0002-3563-9686 


\begin{abstract}
Stoichiometry has been recognized as one of the topics of chemistry that involves a greater difficulty for middle-school and even college students to learn. One of the main causes is the lack of coherence of instructional materials, which often do not consider fundamental aspects such as the prerequisite knowledge required, the multilevel language, and alternative conceptions. To start to overcome the lack of intra-unit coherence in instructional materials, the paper proposes the design of a Hypothetical Learning Program (HLP) to teach this topic. A qualitative methodology is used, with which a two-step systematic process of documentary analysis is developed of a number of articles, book chapters, curricular guidelines and basic standards of national and international competences related to learning progressions and stoichiometry. The first step focuses on elucidating the main theoretical and methodological elements that underlie this design approach, also building the conceptual framework of this research. The second step focuses on the description of the design process of the HLP of the stoichiometry, using as reference the principles of design and the sections that configure the structure of the HLP. The research process resulted in three main contributions: a theoretical framework for HLP from the perspective of design studies, the development of the hlp items that generated the theories of specific domain for the teaching/learning of stoichiometry, and the instructional material in digital format. It is concluded that, nowadays, HLP are considered an emerging paradigm in the field of science education, resulting in a successful heuristic aiming to produce specific domain theories and instructional materials with curricular coherence.
\end{abstract}

Keywords

hypothetical learning progression (HLP); stoichiometry; coherence; great ideas of science

\title{
Resumo
}

A estequiometria tem sido reconhecida como um dos tópicos da química de maior dificuldade para o aprendizado dos alunos do ensino médio e até do ensino universitário. Uma das principais causas é a falta de coerência dos materiais instrucionais, que em muitos casos não consideram aspectos fundamentais como o conhecimento prévio necessário, a linguagem multinível e as concepções alternativas. Como uma proposta para começar a superar a falta de coerência intra-unidade nos materiais de instrução, elaboramos uma Trajetória Hipotética de Aprendizagem (THA) para o ensino desse tópico. Utilizamos uma metodologia de perspectiva qualitativa para desenvolver um processo sistemático de análise documental para um conjunto de artigos, capítulos de livros, diretrizes curriculares e normas básicas de competências nacionais e internacionais relacionadas às trajetórias da aprendizagem e estequiometria, que ocorreu em duas fases. A primeira, focada em elucidar os principais elementos teóricos e metodológicos subjacentes a esse projeto, construindo, além disso, o marco conceitual desta pesquisa. A segunda, focada na descrição do processo de elaboração da THA da estequiometria tomando como referência os princípios de elaboração e as seções que configuram a estrutura da THA. Como resultado, o processo de pesquisa deixou três contribuições principais: marco teórico para a THA a partir da perspectiva de estudos de concepção; desenvolvimento dos itens da THA, criando teorias específicas de domínio para o ensino/ aprendizagem de estequiometria; e o material instrucional em formato digital. Concluímos que, atualmente, as THA são consideradas um paradigma emergente no campo do ensino de ciências, resultando em uma heurística de sucesso com vistas a produzir teorias de domínio específico e materiais instrucionais com coerência curricular.

Palavras-chave

Trajetória Hipotética de Aprendizagem (THA); estequiometria; coerência; grandes ideias da ciência 


\section{Introducción}

En las últimas décadas, los educadores e investigadores del campo de la educación en ciencias han visto con gran preocupación la dificultad de los estudiantes para alcanzar un aprendizaje por comprensión conceptual e integrada de temas específicos. De allí que se hayan buscado alternativas de solución desde distintas perspectivas que a la postre no han impactado las aulas de manera efectiva (Candela, 2016; Nuthall, 1999). Prueba de ello se tiene en los resultados desalentadores de los estudiantes en distintas pruebas estandarizadas para matemáticas y ciencias, como PISA y TIMSS ${ }^{1}$. Se destaca que a partir de este último estudio se ha identificado la coherencia curricular como una de las variables fundamentales que permiten predecir el éxito de los estudiantes en ciencias (Schmidt, Wang y McKnight, 2005).

De hecho, en el diseño colombiano de muchos de los materiales instruccionales de ciencias, llama la atención el elevado número de tenas con profundidad insuficiente y falta de coherencia entre diferentes aspectos de la enseñanza como son: las metas de aprendizaje, las prácticas científicas, el diseño curricular de los contenidos, las estrategias instruccionales, las actividades de aprendizaje y las formas de evaluar las ideas científicas, entre otras (coherencia intraunidad).

Así pues, la problemática curricular recogida por los materiales instruccionales es una consecuencia directa de no tener en cuenta de manera explícita las teorías educativas de perspectivas generales y específicas de las ciencias naturales ${ }^{2}$ durante el proceso de diseño

1 Las pruebas Third International Mathematics And Science Study (TIMSS) fueron realizadas en 1995 para evaluar a más de medio millón de estudiantes de 41 países, en los grados 4, 8 y 12 (o último de la educación media), en las áreas de matemáticas y ciencias naturales.

2 Las teorías generales, o de tamaño de grano grueso, hacen referencia a las teorías del aprendizaje y la pedagogía general. Las teorías intermedias hacen alusión a referentes de tamaño de grano medio, como son los modelos y teorías (p. ej., las teorías del aprendizaje, la pedagogía general, las teorías del diseño instruccional, y la literatura en ciencias). Como alternativa, el diseño de ambientes de aprendizaje de temas específicos informados por esta clase de teorías de naturaleza genérica, le permite al diseñador o profesor establecer de manera sistemática las relaciones entre los elementos de la enseñanza, para superar la falta de coherencia intra e intercurricular (Reigeluth, 1999; Wiley, 2000).

Por otro lado, los estudios de diseño de ambientes de aprendizaje de temas específicos tienen como uno de sus propósitos claves ayudar a disminuir la ruptura existente entre la teoría proveniente de la investigación educativa y la práctica del diseño y la enseñanza (Lagemann, 2002; Levin y O’Donnell, 1999; National Research Council [NRC], 2002). Para ello, el diseñador a lo largo del proceso de diseño toma la decisión de seleccionar los elementos teóricos provenientes de las teorías de naturaleza general y específica que estén alineados con el tema en consideración, con el fin de apoyar la toma de decisiones curriculares e instruccionales, las cuales posteriormente se traducirán en la estructura lógica del material instruccional en mención.

Pasando al plano disciplinar, llama la atención que la compleja situación enunciada hasta ahora, es común para el diseño de ambientes de aprendizaje de los temas de las distintas áreas de las ciencias naturales y tiende a acentuarse en la química. Punto de vista reconocido desde tiempo atrás por distintos educadores e investigadores del campo de la educación en ciencias (Furió, Azcona y Guisasola, 2002). Así pues, las ideas que configuran la estructura lógica de dicha disciplina resultan ser de un nivel alto de complejidad para su comprensión conceptual,

que han dado origen a las progresiones de aprendizaje. Por su parte, las teorías de grano fino son teorías de dominio específico, que recogen la toma de decisiones curriculares e instruccionales de un tema específico. 
destacándose la estequiometría. Naturalmente, esta idea es considerada difícil de internalizar como consecuencia de su alta naturaleza abstracta, la gran cantidad de conceptos que deben relacionarse, la dependencia de conocimientos que son requisito y la exigencia en los estudiantes de asociar y representar la materia así como sus interacciones a partir del lenguaje multinivel (Gabel, 1998).

Como respuesta a la problemática descrita, en las últimas décadas ha emergido desde la línea del diseño instruccional la perspectiva de las progresiones de aprendizaje hipotéticas (HLP). Efectivamente, este instrumento resulta pertinente para el diseño de un material instruccional de la gran idea de la estequiometría, dotado con los atributos necesarios en cuanto a coherencia intraunidad, ya que permite la alineación de elementos esenciales como: los conocimientos que son requisito, las subideas derivadas de los distintos estándares del tema en consideración, las prácticas científicas y las metas de aprendizaje, entre otros.

Tomando en consideración los anteriores presupuestos, este estudio tiene como propósito dar respuesta al siguiente interrogante de investigación: ¿̇Cómo diseñar una progresión de aprendizaje con coherencia curricular, que le permita al estudiante alcanzar una comprensión conceptual e integrada de la gran idea de la estequiometría?

\section{Antecedentes}

En la literatura existente sobre educación en ciencias, no se ha evidenciado la existencia de una progresión de aprendizaje hipotética (HLP) para la enseñanza de la estequiometría. A la fecha, se han desarrollado un limitado número de progresiones de aprendizaje para algunos tópicos específicos relacionados con la química, entre los que se destacan los siguientes:

En primer lugar, el trabajo realizado por Smith, Wiser, Anderson y Krajcik (2006), el cual sienta un precedente sobre el uso de las HLP en el campo de la educación en química, ya que aborda el desarrollo de una progresión de aprendizaje para la enseñanza de la teoría atómica y molecular de la materia. Dicha investigación se centra en la manera en la cual los estudiantes razonan y aprenden en torno a grandes ideas, como base para llegar a un aprendizaje significativo, introduciendo además el concepto de desempeños de aprendizaje. También, la investigación contribuyó en la construcción de la HLP para la estequiometría, al servir como referente en aspectos como el diseño y la secuenciación de las grandes ideas a partir de los estándares de competencias, así como en la elaboración de desempeños de aprendizaje y la evaluación formativa.

Un segundo trabajo, adelantado por Stevens, Delgado y Krajcik (2010) describe los esfuerzos por desarrollar una progresión de aprendizaje hipotética (HLP) para estudiantes de 7 a 14, sobre la estructura, comportamiento y propiedades de la materia, así como sus relaciones a nanoescala, con la ciencia y la ingeniería (NSE). La progresión se construyó a partir de datos empíricos que caracterizan la forma en la cual los estudiantes usualmente desarrollan su conocimiento, como parte de la elaboración y refinamiento de la HLP. En este trabajo se usó como 
metodología el diseño centrado en el constructo (CCD), el cual sirve de referente para el desarrollo de la HLP sobre la estequiometría, ya que considera aspectos como: anclajes inferior y superior, metas de aprendizaje, evidencias y actividades de aprendizaje, así como niveles intermedios.

\section{Marco conceptual}

La investigación basada en el diseño es un paradigma emergente para el estudio del aprendizaje en contextos específicos. Esta clase de investigación es de carácter formativo, y tiene como fin diseñar, implementar y evaluar ambientes de aprendizaje para generar teorías de dominio específico. Su estructura se fundamenta en el diseño sistemático, el estudio de estrategias instruccionales y herramientas, que buscan disminuir la brecha existente entre la investigación en el campo educativo y los problemas de aprendizaje en contextos reales (Brown, 1992; Collins, 1992). Como producto de dicha investigación, han surgido distintas perspectivas de diseño cuyo propósito central es asistir a los estudiantes en la comprensión conceptual e integrada de las grandes ideas dentro y a lo largo de los grados. Entre ellas pueden mencionarse: los corredores conceptuales, las teorías del diseño instruccional, las trayectorias conceptuales (Driver, Leach, Scott y Wood-Robinson, 1994), los corredores de desarrollo (Brown, 1997), las secuencias de enseñanza y aprendizaje (Ametller, Leach y Scott, 2007) y las progresiones de aprendizaje (NRC, 2007). Se destaca que el presente estudio está enmarcado dentro de la perspectiva de las progresiones de aprendizaje a pequeña escala.

Un aspecto clave en el diseño de ambientes de aprendizaje es la coherencia curricular de los materiales instruccionales. De hecho, se le ha considerado uno de los factores determinantes para el éxito de los estudiantes tanto en sus procesos de aprendizaje, como en su desempeño en ciencias naturales en el momento de participar en pruebas estandarizadas, bien sea a nivel nacional o internacional.

En este sentido, la coherencia curricular presupone el avance progresivo de las grandes ideas, dentro de un mismo nivel y a través de los diferentes grados de escolaridad, hacia formas de pensamiento cada vez más sofisticado en la estructura de las disciplinas, alcanzando cada vez mayor profundidad. Específicamente, la coherencia intraunidad se enfoca hacia la alineación entre el contenido de las metas de aprendizaje, las prácticas científicas, las actividades de aprendizaje, las estrategias instruccionales y las formas de evaluación, aspectos centrales en el diseño de la presente HLP.

En cuanto a las progresiones de aprendizaje puede decirse que como una de las perspectivas de los estudios de diseño, se ha empezado a considerar un enfoque de diseño emergente dentro del campo de la educación en ciencias, razón por la cual la literatura existente sobre el tema aparece publicada principalmente a partir del año 2009 (Duschl, 2011 , p. 124). Se destaca que, al igual que los otros enfoques sobre el diseño de ambientes de aprendizaje, este tiene su génesis desde los experimentos de enseñanza y los estudios de entrevista clínica.

A pesar de que aún no existe un consenso entre la comunidad de investigadores sobre la definición de las HLP, puede decirse que son

descripciones de formas sucesivamen-

te más sofisticadas de pensamiento acerca de un tema, el cual forma parte de una secuencia, de acuerdo con el proceso de aprendizaje e investigación por parte de los estudiantes. Dicho proceso puede darse en periodos de tiempo tan extensos como seis u ocho años. (NRC, 2007, p. 213)

En este sentido, se puede afirmar que las progresiones de aprendizaje son estudios de diseño que pretenden integrar los principales componentes del currículum de ciencias, con 
el fin de conseguir una estructura coherente en todas sus dimensiones. De esta manera, buscan dar respuesta a la falta de alineación entre el diseño curricular, los materiales para la enseñanza, la práctica pedagógica y la evaluación.

Entre los principales componentes de las HLP se destaca la existencia de dos tipos de anclaje, uno inferior y otro superior, entre los cuales se halla una serie de niveles intermedios a través de los cuales discurren en secuencia las grandes ideas, sin que por ello deba asociarse con un carácter lineal. Adicionalmente, se incluyen elementos como ideas centrales, estándares de contenido, estándares de indagación, desempeños de aprendizaje y metas de aprendizaje. Entre sus ventajas, puede mencionarse que su estructura permite caracterizar mejor a los estudiantes, razón por la cual resulta pertinente para un estudio de ocho semanas, como es el propósito de la presente propuesta (Cataño, 2016).

Desde una perspectiva epistemológica, la HLP de la estequiometría está fundamentada a partir de marcos teóricos de tres categorías. Unos de tamaño de grano grueso que tienen un carácter explicativo y buscan ilustrar la manera como aprende un sujeto. Otros de tamaño de grano medio relacionados con el origen de las HLP y, finalmente, las herramientas de diseño que también incluyen teorías de tamaño de grano grueso y fino para convertirse en uno de los principales instrumentos que asisten al diseñador instruccional a lo largo del diseño de la HLP. En otras palabras, iluminan la toma de decisiones curriculares e instruccionales (véase tabla 1).

Tabla 1. Marcos teóricos que fundamentan las LP

\begin{tabular}{|l|l|l|}
\hline \multicolumn{1}{|c|}{ Categoría } & \multicolumn{1}{|c|}{ Elementos teóricos } & \multicolumn{1}{c|}{ Contenidos } \\
\hline \multirow{2}{*}{ Tamaño de grano grueso } & Teorías del aprendizaje & $\begin{array}{l}\text { Teoría constructivista cognitiva } \\
\text { (Piaget, 1974) } \\
\text { Teoría constructivista sociocultural } \\
\text { Teoría del procesamiento de la } \\
\text { información }\end{array}$ \\
\cline { 2 - 3 } & Pedagogía general & $\begin{array}{l}\text { Técnicas, estrategias y modelos } \\
\text { de enseñanza }\end{array}$ \\
\hline \multirow{2}{*}{ Tamaño } & $\begin{array}{l}\text { Modelo de reconstrucción } \\
\text { educativa (Duit, Gropengieber y } \\
\text { Kattman, 2005) } \\
\text { Uso del lenguaje }\end{array}$ \\
\hline Tamaño de grano fino & Teorías intermedias & $\begin{array}{l}\text { Ciclo de aprendizaje } \\
\text { Diferenciación en integración de } \\
\text { los niveles de representación en } \\
\text { la química como herramienta de } \\
\text { comunicación (Johnstone, 1982) } \\
\text { La estequiometría como eje } \\
\text { fundamental de la química } \\
\text { cuantitativa } \\
\text { Concepciones alternativas }\end{array}$ \\
\hline
\end{tabular}

Fuente: elaboración propia. 
En lo referente al aspecto disciplinar, la gran idea de la estequiometría constituye uno de los principales tópicos de la química a nivel de educación media. Su aprendizaje, representa un desafío para maestros y estudiantes, dado su alto nivel de complejidad, ya que implica el conocimiento de varios aspectos relacionados con procesos de abstracción demandantes para los aprendices, por ejemplo, el concepto de mol y el esquema de la proporcionalidad. En este sentido, se ha comenzado a considerar que el aprendizaje por comprensión conceptual e integrada de dicha idea, necesita de la articulación tanto en el diseño como en la implementación de los siguientes elementos: contenido de la estequiometría, esquemas de la conservación de propiedades no observables y proporcionalidad y las prácticas científicas (Candela, 2012; Pozo y Gómez, 1998).

Para que los estudiantes logren un aprendizaje por comprensión conceptual e integrada de la gran idea de la estequiometría, deben converger en las actividades de aprendizaje los tres elementos mencionados. Así pues, es necesario que exista una base sólida, cimentada sobre los esquemas conceptuales de la química, para que puedan desarrollarse de manera concomitante los conceptos relacionados con la estequiometría y las prácticas científicas. Esto significa que el estudiante, de manera previa, debe tener un mínimo dominio de esquemas como interacción sistémica, conservación de propiedades no observables, equilibrio y proporcionalidad (Pozo y Gómez, 1998). Dichos esquemas, podrán desarrollarse con mayor profundidad al avanzar a través de las subideas en las que se ha dividido la estequiometría para facilitar su aprendizaje.

Las subideas mencionadas son: (1) cambio químico, (2) ley de la conservación de la masa, (3) reactivo límite, (4) rendimiento de una reacción química y (5) pureza de un reactivo. En el desarrollo de cada una, paso a paso, se presentan nuevos desafíos para los aprendices, que les brindan la oportunidad de avanzar hacia formas más sofisticadas de pensamiento. Esto, gracias a la secuencia y profundidad de los contenidos, donde además se ha tenido en cuenta la edad de los estudiantes, sus concepciones alternativas y sus conocimientos requisito.

\section{Metodología}

Direccionados por el problema de investigación, se ha tomado la decisión de asumir dos fases de recolección y análisis de datos para el diseño sistemático de la HLP. Así, la primera fase tiene como tarea central elucidar los principales elementos teóricos y metodológicos que subyacen a este enfoque de diseño y, además, construir el marco conceptual de esta investigación, para lo cual se ha llevado a cabo un análisis de contenido desde la perspectiva de Krippendorff (1990) haciendo uso de rejillas de análisis. En la segunda fase se realiza una descripción del proceso de diseño de la HLP de la estequiometría tomando como referencia los principios de diseño y las secciones que configuran la estructura de la HIP (p. ej., selección de la gran idea; descomposición de la gran idea en subideas; metas de aprendizaje; diseño, selección, secuenciación y temporalización de actividades de aprendizaje; entre otros), para ello, también se utiliza la técnica de análisis de contenido, pero desde un enfoque inductivo. Es decir que se efectúa una lectura sistemática a las respectivas unidades de contexto, con el fin de obtener información que se encuentre alineada con cada una de las diferentes secciones que configuran la HLP y, de esta forma, construir unas teorías de dominio sobre la enseñanza y aprendizaje de la estequiometría. 
Ahora bien, a lo largo de ambas fases, el análisis de contenido se lleva a cabo por medio de una lectura sistemática, reflexiva, crítica, objetiva, replicable y validada de todo el corpus de información contenido en las unidades de contexto. Así pues, a cada una de dichas unidades se le hace un proceso de codificación abierta y axial (Strauss y Corbin, 2002), con el fin de ordenar conceptualmente la información contenida en las fuentes documentales y generar una teoría que dé solución a la pregunta de investigación.

En la realización del análisis documental se tuvieron en cuenta para la unidad de muestreo los artículos y capítulos de libros, relacionados con las HLP y con la educación en química. Adicionalmente, en la segunda fase debió realizarse una revisión de los lineamientos curriculares y los estándares básicos de competencias nacionales e internacionales en relación con el currículum prescrito. Desde dichos elementos, se concibió la estructura general de la HLP, la cual se divide en cuatro secciones (véase tabla 2).

Tabla 2. Secciones de la progresión de aprendizaje hipotética (HLP)

\begin{tabular}{|l|l|}
\hline \multicolumn{2}{|c|}{ Elementos de la HLP } \\
\hline Sección & \multicolumn{1}{c|}{ Contenido } \\
\hline 1 & Contexto de la progresión de aprendizaje. \\
\hline 2 & $\begin{array}{l}\text { Teorías de nivel de grano grueso que soportan el diseño de la progresión de aprendizaje de } \\
\text { la gran idea de la estequiometría. }\end{array}$ \\
\hline 3 & Análisis conceptual de la gran idea de la estequiometría para propósitos curriculares. \\
\hline & $\begin{array}{l}\text { Secuenciación, temporalización, representación y elección de estrategias pedagógicas de la } \\
\text { gran idea de la estequiometría. }\end{array}$ \\
\hline
\end{tabular}

Fuente: elaboración propia.

\section{Resultados y análisis}

En la presente sección, se explicitan los resultados arrojados como producto del proceso de análisis documental de las distintas unidades de muestreo relacionadas con el diseño de la HLP de la estequiometría. En este sentido, se obtuvieron tres resultados. El primero es el marco teórico de la HLP mencionado arriba; el segundo, las teorías de dominio específico para la enseñanza de la estequiometría que se representan y documentan en una estructura de cinco secciones ${ }^{3}$, y el tercero consistente en el material instruccional informado por las teorías de dominio, que además cuenta con una interfaz ubicada en el sitio http://roosvelt7. wix.com/estequiometria.

3 Por razones de espacio, el formato que registra y representa las teorías de dominio específico de la estequiometría se presenta en este documento por medio de una estructura de texto continuo. Además, no se abordará la sección del contexto sociocultural. 
Tabla 3. Teorías de dominio específico para la enseñanza de la estequiometría

\section{Secuenciación, temporalización, representación y elección de estrategias pedagógicas} de la gran idea de la estequiometría

En esta sección se presentan y justifican, en un nivel de grano fino, las decisiones de diseño sobre la secuenciación y temporalización de las subideas que configuran la estequiometría. Además, se seleccionan y/o diseñan las estrategias pedagógicas y formas de representar y formular dichas ideas, con el fin de brindar la oportunidad a los estudiantes de explicitar las concepciones alternativas, desarrollarlas progresivamente e implementarlas en otros escenarios.

Análisis de la lógica de la disciplina para la enseñanza con fines curriculares

El siguiente apartado recoge los principales componentes de la progresión de aprendizaje para la enseñanza de la estequiometría, discriminados para cada una de las cinco subideas en las cuales se ha secuenciado la enseñanza de la estequiometría.

\begin{tabular}{|c|c|c|}
\hline Estándar de contenido & Estándar de indagación & Desempeño de aprendizaje \\
\hline $\begin{array}{l}\text { (I) Relaciono la estructura de las } \\
\text { moléculas orgánicas e inorgánicas con } \\
\text { sus propiedades físicas y químicas y su } \\
\text { capacidad de cambio químico. }\end{array}$ & $\begin{array}{l}\text { Elaboro el modelo de la reacción } \\
\text { química para explicar, en términos } \\
\text { generales, el proceso de transformación } \\
\text { de reactivos en productos (p. ej., la } \\
\text { combustión del etanol). }\end{array}$ & $\begin{array}{l}\text { Empleo modelos moleculares para } \\
\text { explicar la manera en la cual las } \\
\text { moléculas de los reactivos interactúan } \\
\text { transformándose en otras entidades } \\
\text { moleculares (p. ei., la combustión del } \\
\text { etanol). }\end{array}$ \\
\hline $\begin{array}{l}\text { (II) Establezco relaciones a nivel } \\
\text { submicroscópico, macroscópico y } \\
\text { simbólico, entre las cantidades de } \\
\text { sustancia durante una transformación } \\
\text { química, con el fin de verificar la ley de } \\
\text { la conservación de la masa. }\end{array}$ & $\begin{array}{l}\text { Utilizo el modelo corpuscular de la } \\
\text { materia junto al esquema conceptual } \\
\text { de la proporcionalidad directa, como } \\
\text { elementos para evidenciar la ley de } \\
\text { la conservación de la masa (p. ej., la } \\
\text { oxidación del hierro). }\end{array}$ & $\begin{array}{l}\text { Uso el modelo corpuscular de la } \\
\text { materia junto al esquema conceptual } \\
\text { de la proporcionalidad directa, para } \\
\text { demostrar la ley de la conservación de } \\
\text { la masa, en términos de los tres niveles } \\
\text { de representación de la química (p. ej., } \\
\text { la oxidación del hierro). }\end{array}$ \\
\hline $\begin{array}{l}\text { (III) Interpreto una ecuación química a } \\
\text { nivel macroscópico y submicroscópico, } \\
\text { con el fin de identificar el número } \\
\text { de moléculas, gramos y moles que } \\
\text { intervienen en dicho proceso como } \\
\text { reactivos y productos. Para ello, utilizo } \\
\text { la teoría corpuscular, la mol como } \\
\text { unidad de cantidad de sustancia y la } \\
\text { cantidad de masa expresada en gramos } \\
\text { para calcular el reactivo límite en una } \\
\text { reacción química. }\end{array}$ & $\begin{array}{l}\text { Realizo una práctica científica con } \\
\text { materiales de uso común, en la cual } \\
\text { se calcule la cantidad de reactivos } \\
\text { necesarios para obtener una } \\
\text { cantidad determinada de producto. } \\
\text { Adicionalmente, recojo, organizo y } \\
\text { analizo los datos acerca de las muestras } \\
\text { de reactivos y productos, en el contexto } \\
\text { de la ley de la conservación de la masa } \\
\text { (p. ej., la reacción entre el magnesio } \\
\text { metálico y el ácido clorhídrico). }\end{array}$ & $\begin{array}{l}\text { Interpreto y analizo los datos de los } \\
\text { reactivos y productos en términos } \\
\text { de relaciones de proporcionalidad } \\
\text { en gramos y moles, con el fin } \\
\text { de comprender, tanto a escala } \\
\text { macroscópica como submicroscópica la } \\
\text { importancia del reactivo límite en una } \\
\text { reacción química. }\end{array}$ \\
\hline $\begin{array}{l}\text { (IV) Aplico los conceptos básicos de las } \\
\text { relaciones estequiométricas con el fin de } \\
\text { calcular el rendimiento de una reacción } \\
\text { química. }\end{array}$ & $\begin{array}{l}\text { Explico el rendimiento de una reacción } \\
\text { química a partir de las relaciones } \\
\text { estequiométricas entre los reactivos y los } \\
\text { productos (p. ej., la combustión de la } \\
\text { gasolina). }\end{array}$ & $\begin{array}{l}\text { Utilizo el modelo matemático que } \\
\text { representa el rendimiento de una } \\
\text { reacción química, con el fin de } \\
\text { seleccionar, entre distintos procesos, } \\
\text { aquellas reacciones que son más } \\
\text { productivas en la industria química o en } \\
\text { la vida cotidiana (p. ej., la combustión } \\
\text { de la gasolina en los vehículos). }\end{array}$ \\
\hline $\begin{array}{l}\text { (V) Determino el porcentaje de pureza } \\
\text { de un reactivo, al confrontar la } \\
\text { cantidad de producto que teóricamente } \\
\text { debe formarse, con la obtenida } \\
\text { experimentalmente. }\end{array}$ & $\begin{array}{l}\text { Utilizo las relaciones estequiométricas } \\
\text { entre reactivos y productos para } \\
\text { determinar el porcentaje de pureza de } \\
\text { uno de los reactivos (p. ej., el oro de } 24 \\
\text { y } 18 \text { quilates). }\end{array}$ & $\begin{array}{l}\text { Utilizo el modelo matemático que } \\
\text { representa el cálculo de la pureza de un } \\
\text { reactivo para determinar la cantidad de } \\
\text { impurezas presentes en una muestra de } \\
\text { un reactivo dado. }\end{array}$ \\
\hline
\end{tabular}




\begin{tabular}{|c|c|c|c|c|}
\hline Anclaje inferior & $\begin{array}{l}\text { Gran idea de la } \\
\text { estequiometría }\end{array}$ & $\begin{array}{l}\text { nes } \\
\text { as y } \\
\text { les }\end{array}$ & Meta de aprendizaje & Anclaje superior \\
\hline \multirow{5}{*}{$\begin{array}{l}\text { La materia está } \\
\text { constituida por un gran } \\
\text { número de átomos y } \\
\text { moléculas, los cuales } \\
\text { se encuentran en un } \\
\text { perpetuo movimiento, } \\
\text { además, experimentan } \\
\text { diferentes tipos de } \\
\text { fuerzas. } \\
\text { Los modelos teóricos } \\
\text { de la química se han } \\
\text { construido, comunicado } \\
\text { y validado a través de } \\
\text { un lenguaje multinivel, } \\
\text { que está constituido } \\
\text { por la interacción de } \\
\text { los siguientes niveles } \\
\text { de representación: } \\
\text { macroscópico, } \\
\text { submicroscópico y } \\
\text { simbólico. } \\
\text { El esquema conceptual } \\
\text { de la relación de } \\
\text { proporcionalidad } \\
\text { directa subyace } \\
\text { a la comprensión } \\
\text { conceptual e integrada } \\
\text { de la gran idea de la } \\
\text { estequiometría. } \\
\text { En todas las } \\
\text { transformaciones } \\
\text { químicas, la cantidad } \\
\text { de materia permanece } \\
\text { constante, sin importar } \\
\text { su aspecto final, por } \\
\text { ejemplo: una aparente } \\
\text { reducción del volumen } \\
\text { en los productos. } \\
\text { Los átomos y las } \\
\text { moléculas son modelos } \\
\text { teóricos que nos han } \\
\text { permitido explicar las } \\
\text { transformaciones de la } \\
\text { materia. } \\
\text { Adicionalmente, estos } \\
\text { se pueden contar por } \\
\text { medio de la unidad de } \\
\text { cantidad de sustancia, } \\
\text { la mol, logrando de este } \\
\text { modo vincular el mundo } \\
\text { macroscópico con el } \\
\text { submicroscópico. } \\
\text { Diferenciación e } \\
\text { integración entre } \\
\text { los cambios físicos } \\
\text { y químicos a través } \\
\text { del desarrollo de las } \\
\text { propiedades observables } \\
\text { y no observables (p. } \\
\text { ej., identidad de las } \\
\text { sustancias y conservación } \\
\text { de la masa). }\end{array}$} & $\begin{array}{l}\text { (1) A lo largo de la } \\
\text { reacción química, } \\
\text { la identidad de las } \\
\text { moléculas cambia, } \\
\text { pero la de los } \\
\text { átomos se conserva, } \\
\text { como resultado } \\
\text { del debilitamiento } \\
\text { y fortalecimiento } \\
\text { concomitante de } \\
\text { fuerzas intra e } \\
\text { intermoleculares. }\end{array}$ & \multirow{5}{*}{$\begin{array}{l}\text { En los cálculos } \\
\text { cuantitativos es } \\
\text { común encontrar } \\
\text { que ante la } \\
\text { dificultad de } \\
\text { los estudiantes } \\
\text { para resolver } \\
\text { situaciones } \\
\text { problema, ellos } \\
\text { recurren a distintas } \\
\text { estrategias } \\
\text { que aunque } \\
\text { ocasionalmente } \\
\text { pueden funcionar, } \\
\text { pero suelen } \\
\text { conducir a } \\
\text { respuestas } \\
\text { erróneas. Las } \\
\text { principales son: } \\
\text { la estrategia } \\
\text { cualitativa, la } \\
\text { estrategia aditiva, } \\
\text { la estrategia por } \\
\text { correspondencia } \\
\text { y la estrategia } \\
\text { proporcional } \\
\text { (véase sección } \\
2.2 .1 .1 .2 \text { ). En } \\
\text { general, estas } \\
\text { surgen de aplicar } \\
\text { ciegamente } \\
\text { algoritmos, sin } \\
\text { comprender } \\
\text { realmente los } \\
\text { procesos que } \\
\text { están llevando } \\
\text { a cabo (Pozo y } \\
\text { Gómez, 1998). }\end{array}$} & $\begin{array}{l}\text { Nivel exploratorio: Identifico cambios } \\
\text { químicos en la vida cotidiana y en el } \\
\text { ambiente. } \\
\text { Nivel descriptivo: Identifico la formación de } \\
\text { nuevas sustancias como una consecuencia } \\
\text { de la reorganización de los átomos o las } \\
\text { moléculas, debido al debilitamiento o } \\
\text { fortalecimiento de fuerzas intermoleculares. } \\
\text { Comparo mecanismos de obtención de } \\
\text { energía en los seres vivos (6-7). } \\
\text { Nivel explicativo: explico algunos cambios } \\
\text { químicos que ocurren en el ser humano. }\end{array}$ & \multirow{5}{*}{$\begin{array}{l}\text { Con base en } \\
\text { principios como las } \\
\text { leyes ponderales, } \\
\text { y las relaciones } \\
\text { cuantitativas entre } \\
\text { las especies de una } \\
\text { ecuación química } \\
\text { balanceada, } \\
\text { es posible } \\
\text { realizar cálculos } \\
\text { relacionados } \\
\text { con la masa de } \\
\text { cualquiera de sus } \\
\text { componentes, } \\
\text { con solo saber la } \\
\text { cantidad de materia } \\
\text { presente en alguno } \\
\text { de los reactivos } \\
\text { o productos. } \\
\text { Ejercicio que } \\
\text { puede realizarse, } \\
\text { tanto en el plano } \\
\text { teórico como en el } \\
\text { experimental. } \\
\text { En el plano } \\
\text { teórico es posible } \\
\text { determinar } \\
\text { la masa de } \\
\text { producto formado } \\
\text { considerando } \\
\text { determinadas } \\
\text { condiciones } \\
\text { de pureza de } \\
\text { los reactivos o } \\
\text { teniendo en cuenta } \\
\text { el porcentaje de } \\
\text { rendimiento de un } \\
\text { sistema. }\end{array}$} \\
\hline & $\begin{array}{l}\text { (2) La ley de la } \\
\text { conservación de } \\
\text { la masa permite } \\
\text { establecer que la } \\
\text { cantidad de átomos } \\
\text { de cada elemento, } \\
\text { debe coincidir al } \\
\text { comparar reactivos } \\
\text { con productos. }\end{array}$ & & $\begin{array}{l}\text { Nivel exploratorio: identifico la ley de la } \\
\text { conservación de la masa como fundamento } \\
\text { del concepto de estequiometría. } \\
\text { Nivel descriptivo: establezco el balance } \\
\text { de la cantidad de materia entre reactivos } \\
\text { y productos para una ecuación química, } \\
\text { mediante los coeficientes estequiométricos } \\
\text { apropiados. } \\
\text { Nivel explicativo: predigo la cantidad de } \\
\text { masa que debe haber en los productos, a } \\
\text { partir de la masa de los reactivos. }\end{array}$ & \\
\hline & $\begin{array}{l}\text { (3) El reactivo límite } \\
\text { permite establecer } \\
\text { relaciones de } \\
\text { proporcionalidad } \\
\text { directa entre } \\
\text { reactivos para } \\
\text { determinar las } \\
\text { cantidades de los } \\
\text { productos dentro } \\
\text { de una ecuación } \\
\text { química. }\end{array}$ & & $\begin{array}{l}\text { Nivel exploratorio: identifico el reactivo límite } \\
\text { como referente para la realización de cálculos } \\
\text { relacionados con la cantidad de producto } \\
\text { formado en una reacción química. } \\
\text { Nivel descriptivo: calculo la cantidad de } \\
\text { moles de reactivo necesarios para obtener } \\
\text { determinada cantidad de producto. } \\
\text { Nivel explicativo: calculo el reactivo límite } \\
\text { para distintas tareas problema, a partir de } \\
\text { una ecuación balanceada, bien sea que estén } \\
\text { expresadas en moles o en gramos. }\end{array}$ & \\
\hline & $\begin{array}{l}\text { (4) Las relaciones } \\
\text { estequiométricas } \\
\text { establecidas } \\
\text { entre reactivos } \\
\text { y productos, } \\
\text { permiten calcular el } \\
\text { rendimiento de una } \\
\text { reacción química al } \\
\text { comparar la masa } \\
\text { de los productos } \\
\text { que se obtienen } \\
\text { teóricamente, } \\
\text { con los valores } \\
\text { experimentales. }\end{array}$ & & $\begin{array}{l}\text { Nivel exploratorio: identifico los factores que } \\
\text { afectan la transformación de la totalidad de } \\
\text { las moléculas de reactivos en productos en } \\
\text { una reacción química. } \\
\text { Nivel descriptivo: a partir de la ecuación } \\
\text { balanceada para una reacción química, } \\
\text { determino el porcentaje de rendimiento, } \\
\text { comparando la cantidad de producto formado } \\
\text { según datos de tipo teórico y experimental. } \\
\text { Nivel explicativo: determino el porcentaje } \\
\text { de rendimiento en situaciones problema, } \\
\text { conociendo la masa de reactivos y productos, } \\
\text { tanto real como teórica, de acuerdo con ley } \\
\text { de la conservación de la masa. }\end{array}$ & \\
\hline & $\begin{array}{l}\text { (5) Para un sistema } \\
\text { en equilibrio es } \\
\text { posible determinar } \\
\text { el porcentaje de } \\
\text { pureza de uno de } \\
\text { los reactivos, si se } \\
\text { conoce el porcentaje } \\
\text { de rendimiento } \\
\text { en las mismas } \\
\text { condiciones de } \\
\text { reacción. }\end{array}$ & & $\begin{array}{l}\text { Nivel exploratorio: reconozco que existen } \\
\text { factores que afectan el rendimiento de una } \\
\text { reacción química. } \\
\text { Nivel descriptivo: identifico la pureza de } \\
\text { un reactivo como una de las variables que } \\
\text { afectan el rendimiento de una reacción } \\
\text { química. } \\
\text { Nivel explicativo: calculo la masa de producto } \\
\text { formado en una reacción, teniendo en cuenta } \\
\text { la presencia de un reactivo límite, el efecto del } \\
\text { rendimiento de la reacción y la pureza de los } \\
\text { reactivos. }\end{array}$ & \\
\hline
\end{tabular}

Fuente: elaboración propia. 


\section{Material instruccional}

Las teorías de dominio específico que configuran la HLP informaron el proceso de diseño y desarrollo del material instruccional para la enseñanza de la gran idea de la estequiometría. En efecto, a partir de los presupuestos de dichas teorías, fue posible diseñar una interfaz que recoge las cinco subideas de la estequiometría (http://roosvelt7.wix.com/estequiometria) ${ }^{4}$, cada una de ellas discriminada en las fases de exploración, descripción y explicación; estas fases le permiten al estudiante recorrer el corredor conceptual de la estequiometría de manera progresiva. Adicionalmente, la arquitectura de dicha herramienta tecnológica fue concebida para brindar la posibilidad a los aprendices de alcanzar una comprensión conceptual e integrada del tema en consideración con actividades de aprendizaje.

\section{Conclusiones}

Si bien, las teorías del aprendizaje tienen un carácter descriptivo en la medida en que buscan explicar la manera en la cual los individuos aprenden, también contribuyen a construir teorías prescriptivas como las teorías de dominio específico. Estas últimas tienen la facultad de direccionar la elección de los métodos instruccionales que se pueden emplear para el logro de metas de aprendizaje determinadas. De allí que las HLP resulten útiles como herramientas para orientar las rutas que permiten a los aprendices alcanzar niveles progresivamente más sofisticados de comprensión de las grandes ideas de la ciencia.

4 El material instruccional para la enseñanza de la estequiometría, en su versión digital, cuenta con un sitio Web diseñado exclusivamente para apoyar los procesos de comprensión conceptual e integrada de la gran idea de la estequiometría.
En cuanto a explicitar, documentar y representar a través de la HLP la serie de teorías de dominio específico que se encuentran alineadas con la gran idea de la estequiometría, se llegó a las siguientes conclusiones:

En este estudio de diseño, para ayudar a solucionar el problema de la enseñanza de la estequiometría en la escuela secundaria, se seleccionaron de manera deliberada una serie de elementos teóricos y metodológicos provenientes de la literatura basada en la investigación. Así pues, se indagó en los cuerpos de conocimientos que constituyen las teorías derivadas de la investigación educativa (p. ej., teorías del aprendizaje, pedagogía general, modelo de reconstrucción educativa., uso del lenguaje desde la perspectiva de Vygotsky y Bajtín, niveles de representación de la química, relación sinérgica entre los elementos que componen la estequiometría), con el fin de sustentar la toma de decisiones curriculares e instruccionales que le brindarían la posibilidad al estudiante de alcanzar un aprendizaje por comprensión conceptual e integrada de la gran idea de la estequiometría.

Por tanto, al conjunto de proposiciones que sustentan la toma de decisiones curriculares e instruccionales para el aprendizaje de la estequiometría se le denomina teorías de dominio específico o teorías humildes, las cuales se encuentran estrechamente vinculadas con la gran idea en cuestión. Desde luego que una característica inherente de estas teorías hace referencia al establecimiento de una relación coherente entre los elementos de la enseñanza, tales como: esquemas conceptuales de la química, gran idea de la estequiometría, conceptos transversales y prácticas científicas; situación que le confiere una coherencia intracurricular a la HLP de la estequiometría. 
Por otro lado, estas teorías desempeñan un papel crítico durante el desarrollo del material instruccional, considerando que informan e iluminan la materialización del conjunto de actividades de aprendizaje debidamente seleccionadas, secuenciadas y temporalizadas.

Para representar y documentar la teoría que funda tanto la toma de decisiones curriculares e instruccionales, como a las intenciones de diseño, se construyó un formato a partir de los elementos de diseño propios de los enfoques relacionados con la línea de investigación de los estudios de diseño. De esta manera, ha sido posible representar el contenido teórico de la HLP para la enseñanza-aprendizaje de la estequiometría. Dicha contribución al campo del diseño instruccional, sienta un precedente dentro de la línea en mención, lo cual constituye un potencial referente para el desarrollo de trabajos similares en un futuro.

\section{Referencias}

Ametller, L., Leach, J. y Scott, P. (2007). Using perspectives on subject learning to inform the design of subject teaching: An example from science education. The Curriculum Journal, 18(4), 479-492.

Brown, A. L. (1992). Design experiments: Theoretical and methodological challenges in creating complex interventions in classroom settings. The Journal of the Learning Sciences, 2(2), 141-178.

Brown, A. L. (1997). Transforming schools in to communities of thinking and learning about serious matters. American Psychologist, 52, 399-413.

Candela, B. F. (2012). La captura, la documentación y la representación del cpc de un profesor experimentado y "ejemplar" acerca del núcleo conceptual de la discontinuidad de la materia. (Tesis de Maestría en Educación, Enseñanza y Ciencias). Instituto de Educación y Pedagogía, Universidad del Valle, Santiago de Cali.

Candela, B. F. (2016). La ciencia del diseño educativo. Cali: Universidad del Valle.

Cataño, R. (2016). Diseño de una progresión de aprendizaje hipotética con coherencia curricular para la enseñanza de la estequiometría por comprensión conceptual e integrada. (Tesis de maestría). Universidad del Valle, Santiago de Cali.

Collins, A. (1992). Toward a design science of education. En E. Scanlon y O'Shea (eds.), New directions in educational technology. Nueva York: Springer-Verlag.

Driver, R., Leach, J., Scott, P., y Wood-Robinson, C. (1994). Young people's understanding of science concepts: Implications of cross-age studies for curriculum planning. Studies in Science Education, 24, 75-100. 
Duschl, R., Maenga, S. y Sezen, A. (2011). Learning progressions and teaching sequences: A review and analysis, Studies in Science Education, 47(2), 123-182.

Duit, R., Gropengieber, H. y Kattman, U. (2005). Towards science education research that is relevant for improving practice: The model of educational reconstruction. En H. Fisher (ed.), Developing standards in research on science education: The Esera Summer School 2004. Nueva York: Taylor \& Francis.

Furió, C., Azcona, R., y Guisasola, J. (2002). The learning and teaching of the concepts amount of substance and mole: A review of the literature. Chemistry Education: Research and Practice in Europe, 3(3), 277-292.

Gabel, D. (1998). Complexity of chemistry and implications for teaching. En B. J. Fraser y K. Tobin (eds.), International handbook of science education research. Dordrecht: Kluwer Academic Publishers.

Johnstone, A. H. (1982). Macro and microchemistry. School Science Review, 64, 377-379.

Krippendorff, K. (1990). Metodología de análisis de contenido. Teoría y práctica. México: Paidós.

Lagemann, E. C. (2002). An elusive science: The troubling history of education research. Chicago: University of Chicago Press.

Levin, J. R. y O'Donnell, A. M. (1999). What to do about educational research's credibility gaps? Issues in Education, 5(2), 177-229.

National Research Council (NRC). (2002). Scientific research in education. Committee on Scientific Principles for Education Research, (Shavelson, R. J. y Towne, L., eds.). Washington, DC: The National Academies Press.
National Research Council (NRC). (2007). Taking science to school: Learning and teaching science in grades K-8. (Duschl, R. A., Schweingruber, H. A., y Shouse, A. W., eds.). Washington, DC: The National Academies Press.

Nuthall, G. (1999). The way students learn: Acquiring knowledge from an integrated science and social studies unit. Elementary School Journal, 99, 303-34 1.

Piaget, J. (1974). The child and reality: Problems of genetic psychology (A. Rosin, trad.). Londres: Frederick Miller.

Pozo, J. I. y Gómez, M. A. (1998). Aprender y enseñar ciencia. Madrid: Morata.

Reigeluth, C. M. (1999). What is instructional design theory and how is it changing? En Instructional design theories and models (vol. ॥, pp. 4-29). Mahwah: Lawrence Erlbaum Associates, Publishers.

Schmidt, W. H., Wang, H. C. y Mcknight, C. C. (2005). Curriculum coherence: An examination of US mathematics and science content standards from an international perspective. Journal of Curriculum Studies, 37(5), 525-559.

Smith, C., Wiser, M., Anderson, C. y Krajcik, J. (2006). Implications of research on children's Learning for assessment: Matter and atomic molecular theory. Measurement: Interdisciplinary Research and Perspectives, 4, 11-98.

Stevens, S. Y., Delgado, C. y Krajcik, J. (2010). Developing a hypothetical multi-dimensional learning progression for the nature of matter. Journal of Research in Science Teaching, 6(47), 687-715. 
Strauss, A. y Corbin, J. (2002). Bases de la Investigación Cualitativa: Técnicas y procedimientos para desarrollar la teoría fundamentada. (E. Zimmerman, trad.). Medellín: Facultad de Enfermería de la Universidad de Antioquía. [Trabajo original publicado en 1990]

Wiley, D. A. (2000). Learning object design and sequencing theory. (Disertación doctoral), Brigham Young University. Recuperado de http://davidwiley.com/papers/ dissertation/dissertation.pdf

\section{Para citar el artículo}

Candela B. y Cataño R. (2019). Diseño de una progresión de aprendizaje hipotética para la enseñanza de la estequiometría por comprensión conceptual e integrada. Tecné, Episteme y Didaxis: TED, 45, 107-120. 